\title{
Uso de la flora tradicional de la Reserva de la Biósfera El Cielo, Tamaulipas
}

\author{
Use of the traditional flora of El Cielo Biosphere Reserve, \\ Tamaulipas
}

\begin{abstract}
Sergio Guillermo Medellín Morales' ${ }^{1}$ Ludivina Barrientos Lozano', Silvia del Amo Rodríguez², Pedro Almaguer Sierra', Sandra Grisell Mora Ravelo ${ }^{*}$

Medellín Morales, S. G., Barrientos Lozano, L., Del Amo Rodríguez, S., Almaguer Sierra, P., Mora Ravelo, S. G. Uso de la flora tradicional de la Reserva de la Biósfera El Cielo, Tamaulipas. Investigación y Ciencia de la Universidad Autónoma de Aguascalientes. Número 69: 32-38, septiembre-diciembre 2016.
\end{abstract}

\section{RESUMEN}

La investigación se realizó en los ejidos Alta Cima y San José, Tamaulipas, desde diciembre de 2012 hasta marzo de 2014. El objetivo de la investigación fue determinar la riqueza de conocimiento etnobotánico de los habitantes locales por rangos de edad y por género. Se realizaron entrevistas al azar a 30\% de los hogares; entrevistas a informantes de calidad y dos talleres participativos comunitarios. Se identificaron 156 plantas útiles y 62 familias botánicas, que representan $21 \%$ de la diversidad vegetal de la reserva. El grado de conocimiento en ambas comunidades es similar. La mayoría de las especies útiles son nativas en ambas comunidades, aunque hay mayor porcentaje de especies exóticas demandadas en San José. Se planteó la hipótesis de que aquellos informantes de mayor rango de edad y las mujeres son quienes poseen mayor conocimiento etnobotánico en las comunidades en estudio.

Palabras clave: área natural protegida, bosque de niebla, comunidades campesinas, etnobotánica.

Keywords: natural protected area, cloud forest, peasant communities, ethnobotany.

\section{Recibido: 31 de julio de 2015, aceptado: 2 de junio de 2016}

Instituto Tecnológico de Ciudad Victoria.

2 Centro de Ecoalfabetización y Diálogo de Saberes, Universidad Veracruzana.

3 Instituto de Ecología Aplicada, Universidad Autónoma de Tamaulipas.

* Autor para correspondencia: sgmede@gmail.com
ABSTRACT

The research was conducted in the human settlements Alta Cima and San Jose, from December 2012 to March 2014. The main objective of the research was to determine the affluence of local people ethnobotanical knowledge by age and gender. Random interviews were performed in $30 \%$ of households; as well as quality informant interviews and two participatory community workshops. One hundred and fifty six useful plants and 62 botanical families, which represent $21 \%$ of plant diversity of the reserve, were identified. The degree of knowledge in both communities is similar. Most of the useful species are native in both communities, although there is a higher percentage of exotic species used in San Jose. The hypothesis raised was that elder and women local informants possess greater ethnobotanical knowledge at the studied communities.

\section{INTRODUCCIÓN}

En un sitio biodiverso, como el bosque de niebla de la Reserva de la Biósfera El Cielo (RBEC), que contiene $10 \%$ de la biodiversidad vegetal del país, es importante realizar estudios etnobotánicos que ayuden a comprender las formas de manejo, conocimientos y valores que las personas de estas comunidades construyen en torno al mismo. El objetivo de la investigación fue determinar la riqueza de conocimiento etnobotánico de los habitantes locales por rangos de edad y por género. Los 
patrones de uso y manejo de la flora en ambientes montañosos suponen procesos de interacción entre sistemas sociales y ecosistemas naturales, se adaptan a los ciclos productivos de los mismos, a los nichos ecológicos resultantes de la verticalidad montañosa y a las transformaciones sociales del momento pasando de una generación a otra bajo una dinámica de transferencia y acumulación (Stringer et al., 2006).

Para el caso de la RBEC se han realizado diversos estudios, como los de Lara (1989), Hernández Sandoval et al. (1991), Mora López y Medellín Morales (1991), Garza et al. (2005), González Romo y Gispert (2005), Mora Olivo et al. (2005), Pérez Quilantán et al. (2005), Berrones Benítez y Medellín Morales (2007) y Medellín Morales y Osorio (2007). Solamente dos trabajos específicos tratan sobre la diversidad etnobotánica en la zona de estudio; el de González Romo y Gispert (2005) y el de Pérez Quilantán et al. (2005). En 1985, el decreto de la RBEC como área natural protegida incentivó el uso y manejo de las especies no maderables (Gobierno de Tamaulipas y TNC, 2011). Se plantea la hipótesis de que los informantes de mayor rango de edad y las mujeres poseen los mayores niveles de conocimiento sobre plantas en las comunidades en estudio.

\section{MATERIALES Y MÉTODOS}

La investigación se realizó en los ejidos Alta Cima y San José, municipio de Gómez Farías, Tamaulipas, México. Para obtener la información en campo se realizaron entrevistas semiestructuradas (Hoffman y Gallaher, 2007) a 30\% de las unidades familiares (Alexiades, 1996) con el apoyo de la Ficha de Encuesta Etnobotánica Estandarizada según el modelo utilizado por el programa TRAMIL (Germosen Robineau, 1995) 4; en la siguiente fase se seleccionó un grupo de 10 informantes de calidad para corroborar y/o mejorar la información recabada en la etapa previa a través de recorridos etnobotánicos por áreas de acción cotidiana, elaborando miniherbarios y/o realizando registro fotográfico, materiales usados para animar la discusión en los grupos focales (Arias y Cárdenas López, 2007) al final de cada sesión (Hersch Martínez y González Chévez, 1996).

\footnotetext{
En la Ficha de Encuesta Etnobotánica se solicitaron datos personales (nombre, edad, sexo, escolaridad, lugar de nacimiento propio y de sus padres, actividad a la que se dedica) y los relativos a las plantas útiles (nombre local, forma biológica, lugar de obtención, época de obtención, partes usadas y tipos de uso).
}

Las muestras botánicas recolectadas fueron prensadas, secadas e identificadas según la técnica estándar (Alexiades, 1996) e incorporadas al Herbario del Instituto Tecnológico de Ciudad Victoria (ITCV); para la determinación se utilizaron claves taxonómicas como Flora de Veracruz, Flora del Bajío y de Regiones Adyacentes y Flora de Guatemala, así como cotejo con los ejemplares del Herbario del ITCV. La información etnobotánica fue recopilada en tres periodos: desde diciembre de 2012 hasta noviembre de 2013 (encuestas etnobotánicas); desde diciembre de 2013 hasta marzo de 2014 (caminatas etnobotánicas) y desde abril hasta septiembre de 2014 (grupos focales y talleres participativos). El análisis de la información incluyó el cálculo de los siguientes índices (Toscano González, 2006):

Índice de riqueza: riqueza de conocimiento que tiene una persona sobre las posibilidades de uso de flora en su región.

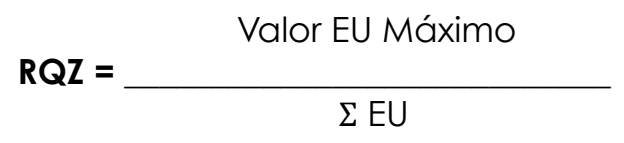

en donde $(R Q Z)=$ riqueza de conocimiento que tiene una persona de las especies útiles en relación con todas las especies útiles encontradas en la región; $(E U)=$ número de especies útiles registradas por una persona; (Valor EU Máximo)= total de especies útiles reportadas en la región por todas las personas participantes del estudio. El valor de este índice varía entre 0 y 1 , donde 1 es el valor máximo de conocimiento de la biodiversidad útil de la región.

Diversidad de uso: cuantificación del número de especies vegetales utilizadas por categorías de uso, así como análisis de la relación de especies exóticas y nativas que componen cada categoría de uso. Se utiliza el índice de uso de especies exóticas (EXÓT) vs. especies nativas (NAT), que hace referencia a la importancia de uso que tienen las especies nativas en relación con las especies exóticas o foráneas para cada usuario. Se calcula así:

NAT $=\frac{\sum \text { EU Nat }}{\Sigma \text { EU }}$
EXG $=\frac{\sum \text { EUExót }}{\Sigma \text { EU }}$


donde $(E U$ Nat)= número de especies útiles nativas reportadas por una persona; $(E U)=$ número de especies útiles usadas por una persona; (EU Exót)= número de especies útiles exóticas reportadas por una persona. Estos valores se comparan para determinar cuál índice es mayor para cada persona, se indica cuáles especies son las más importantes para el mismo. La suma de valores de NAT y EXÓT siempre será 1 .

Para el análisis estadístico se compararon las medias del índice de riqueza en cada localidad con la prueba $t$ de Student.

\section{RESULTADOS}

\section{Descripción de las comunidades}

Ambas comunidades se encuentran ubicadas en el cinturón de bosque de niebla de la RBEC, entre los 900 y 1,300 m.s.n.m., en una porción de la Sierra Madre Oriental conocida como Sierra de Guatemala. La RBEC tiene una extensión de $144,530.51$ ha y está situada al suroeste del estado de Tamaulipas (Gobierno de Tamaulipas y TNC, 2011). Son similares en cuanto a la estructura y composición poblacional (120 en Alta Cima y 100 en San José). En ambas comunidades se presenta una mezcla heterogénea respecto al origen étnico de sus habitantes: habitantes locales y emigrantes (o sus descendientes) de los estados de Michoacán, Hidalgo y Estado de México.

Las principales actividades productivas son la agricultura de temporal (maíz, frijol, calabaza, nopal verdura y cúrcuma) y el manejo de huertos familiares con frutales (durazno y guayaba). Los principales ingresos provienen de la extracción de hojas de palmilla (Chamaedorea radicalis Mart.) y, recientemente, prestación de servicios ecoturísticos por cooperativas campesinas (Gobierno de Tamaulipas y TNC, 2011).

\section{Los habitantes locales y la diversidad de conocimientos}

Los campesinos que participaron en el estudio fueron 20 hombres y 20 mujeres, con promedio de edad de 45 y 42 años, respectivamente. El $70 \%$ de ellos son oriundos de la región y han vivido toda su vida allí; el

5 La mayoría llegó a la región en la época de 1950-1960 cuando los aserraderos estaban en boga en la RBEC. Estas empresas contrataban personas que "iban siguiendo" a los aserraderos.

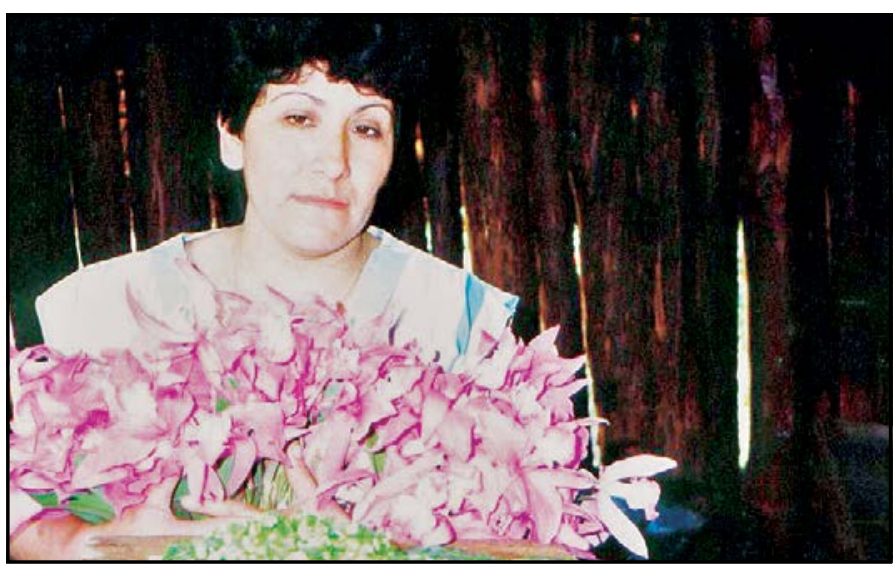

Figura 1. Mujer campesina mostrando dos plantas útiles del bosque de niebla de la RBEC: flor de mayo (Laelia speciosa (Kunth) Schlt.) y nopal (Nopalea cochenillifera L.) Salm-Dyck. Fotografía tomada por los autores.

otro $30 \%$ son inmigrantes de otras regiones serranas del país $5^{5}$ con una historia de ocupación del área de al menos 40 años. El total de especies vegetales útiles en las comunidades estudiadas fue de 156 taxa. Los valores obtenidos mediante la aplicación del índice de riqueza (RQZ) no muestran diferencias significativas (Tabla 1) en el acervo de conocimiento sobre las plantas.

Tabla 1. Prueba $t$ de Student de las medias de plantas útiles por localidad

\begin{tabular}{|l|c|c|}
\hline & Alta Cima & San José \\
\hline Media & 28.5 & 26.6 \\
\hline Varianza & 22.1578947 & 11.2 \\
\hline Observaciones & 20 & 20 \\
\hline Varianza agrupada & 16.6789474 & \\
\hline $\begin{array}{l}\text { Diferencia hipotética de las } \\
\text { medias }\end{array}$ & 0 & \\
\hline Grados de libertad & 38 & \\
\hline Estadístico $\dagger$ & 1.47119175 & \\
\hline$P(T<=\dagger)$ una cola & 0.0747363 & \\
\hline Valor crítico de $t$ (una cola) & 1.68595446 & \\
\hline$P(T<=\dagger)$ dos colas & 0.1494726 & \\
\hline Valor crítico de $\dagger$ (dos colas) & 2.02439416 & \\
\hline
\end{tabular}

En Alta Cima los usuarios conocen $75 \%$ de las 156 especies identificadas para la zona de estudio y en San José los usuarios conocen 63\% (Tabla 2). 
Tabla 2. Índices de conocimiento (RQZ) en las comunidades en estudio

\begin{tabular}{|c|c|c|c|c|c|}
\hline Comunidad & $\begin{array}{c}\text { Taxa } \\
\text { útiles }\end{array}$ & $\begin{array}{c}\text { Taxa } \\
\text { nativas }\end{array}$ & $\begin{array}{c}\text { Taxa } \\
\text { exóticas }\end{array}$ & RQZ Total & $\begin{array}{c}\% \\
\text { Conocim }\end{array}$ \\
\hline Alta Cima & 117 & 65 & 52 & 0.75 & $75 \%$ \\
\hline San José & 98 & 42 & 59 & 0.63 & $63 \%$ \\
\hline Ambas comunidades & 156 & 97 & 59 & & \\
\hline
\end{tabular}

Los valores promedio de conocimiento (RQZ) en ambas comunidades es similar; sin embargo, se identifica el valor más alto (0.33) y más bajo (0.18) en Alta Cima. Los datos muestran que cada persona es poseedor de una porción del conocimiento integral de la región y que, posiblemente, esté relacionado con su estrategia de vida. Los habitantes locales continúan apostándole a la agricultura de subsistencia y a otras alternativas de aprovechamiento integral del bosque (sobre todo de especies no maderables). En la información proporcionada por los campesinos entrevistados hubo marcadas diferencias, el total de los informantes identifican y usan entre 18 y $34 \%$ de las plantas encontradas en la zona ocupada por ambas comunidades.

Al relacionar el índice de conocimiento (RQZ) con los rangos de edad, los valores más altos se encontraron entre los usuarios de edad mediana, tal vez debido a que son personas que están más activas en las labores agrícolas y en el aprovechamiento del bosque (sobre todo de la palmilla). Al relacionar el $R Q Z$ con los rangos de edad a nivel de ambas zonas de estudio, en Alta Cima los mayores valores de RQZ se encuentran en el grupo etario de 41 a 50 años de edad, mientras que en San José corresponden a los de mayor edad (61 y más). Cuando observamos lo que sucede con la riqueza de conocimientos de plantas nativas y exóticas encontramos que el grupo etario que más sabe de plantas nativas, pero menos de exóticas, es el de 41 a 50 años de edad en Alta Cima (Tabla 3).

En lo que respecta al nivel de conocimiento etnobotánico por género, según los datos aportados (Tabla 4) a nivel general, los hombres de Alta Cima poseen un mayor acervo que las mujeres; mientras que en San José estos valores son similares para

Tabla 3. Grupos etarios y riqueza de conocimiento (RQZ) en las comunidades estudiadas

\begin{tabular}{|c|c|c|c|c|c|c|c|c|c|c|c|c|}
\hline \multirow[b]{2}{*}{$\begin{array}{l}\text { Rangos } \\
\text { de edad }\end{array}$} & \multicolumn{6}{|c|}{ ALTA CIMA } & \multicolumn{6}{|c|}{ SAN JOSÉ } \\
\hline & $\begin{array}{l}\text { \# spp. } \\
\text { prom }\end{array}$ & $\begin{array}{l}\text { RQZ } \\
\text { prom }\end{array}$ & $\begin{array}{c}\# \\
\text { spp. } \\
\text { nat }\end{array}$ & $\begin{array}{l}\text { RQZ } \\
\text { nat }\end{array}$ & $\begin{array}{c}\text { \# } \\
\text { spp. } \\
\text { exót }\end{array}$ & $\begin{array}{l}R Q Z \\
\text { exót }\end{array}$ & $\begin{array}{l}\text { \# spp. } \\
\text { prom }\end{array}$ & $\begin{array}{l}\text { RQZ } \\
\text { prom }\end{array}$ & $\begin{array}{c}\# \\
\text { spp. } \\
\text { nat }\end{array}$ & $\begin{array}{l}\text { RQZ } \\
\text { nat }\end{array}$ & $\begin{array}{c}\# \\
\text { spp. } \\
\text { exót }\end{array}$ & $\begin{array}{l}\text { RQZ } \\
\text { exót }\end{array}$ \\
\hline $20-30$ & 25 & 0.21 & 11 & 0.16 & 14 & 0.27 & 26 & 0.26 & 14 & 0.33 & 12 & 0.21 \\
\hline $31-40$ & 27 & 0.23 & 16 & 0.24 & 12 & 0.22 & $v$ & 0 & 0 & 0 & 0 & 0 \\
\hline $41-50$ & 31 & 0.27 & 23 & 0.35 & 8 & 0.16 & 28 & 0.28 & 20 & 0.47 & 8 & 0.14 \\
\hline $51-60$ & 27 & 0.23 & 21 & 0.32 & 6 & 0.11 & 24 & 0.24 & 14 & 0.33 & 10 & 0.18 \\
\hline $61 y+$ & 29 & 0.24 & 15 & 0.23 & 14 & 0.27 & 29 & 0.29 & 18 & 0.43 & 10 & 0.19 \\
\hline
\end{tabular}

Alta Cima: 117 spp. (65 nativas y 52 exóticas); San José: 98 spp. (42 nativas y 56 exóticas).

Tabla 4. Riqueza de conocimiento (RQZ) por género en las comunidades estudiadas

\begin{tabular}{|c|c|c|c|c|c|c|c|c|c|c|c|c|}
\hline \multirow[b]{2}{*}{$\begin{array}{l}\text { Rangos } \\
\text { de edad }\end{array}$} & \multicolumn{6}{|c|}{ HOMBRES (H) } & \multicolumn{6}{|c|}{ MUJERES (M) } \\
\hline & $\begin{array}{l}\text { \# spp. } \\
\text { prom }\end{array}$ & $\begin{array}{l}\text { RQZ } \\
\text { prom }\end{array}$ & $\begin{array}{c}\# \\
\text { spp. } \\
\text { nat }\end{array}$ & $\begin{array}{l}\text { RQZ } \\
\text { nat }\end{array}$ & $\begin{array}{c}\text { \# } \\
\text { spp. } \\
\text { exót }\end{array}$ & $\begin{array}{l}\text { RQZ } \\
\text { exót }\end{array}$ & $\begin{array}{l}\text { \# spp. } \\
\text { prom }\end{array}$ & $\begin{array}{l}\text { RQZ } \\
\text { prom }\end{array}$ & $\begin{array}{c}\text { \# } \\
\text { spp. } \\
\text { nat }\end{array}$ & $\begin{array}{c}\text { RQZ } \\
\text { nat }\end{array}$ & $\begin{array}{c}\text { \# } \\
\text { spp. } \\
\text { exót }\end{array}$ & $\begin{array}{l}\text { RQZ } \\
\text { exót }\end{array}$ \\
\hline $\begin{array}{l}\text { Alta } \\
\text { Cima }\end{array}$ & 29 & 0.25 & 19 & 0.29 & 10 & 0.19 & 28 & 0.24 & 16 & 0.25 & 11 & 0.22 \\
\hline San José & 27 & 0.27 & 18 & 0.44 & 8 & 0.15 & 26 & 0.27 & 15 & 0.35 & 11 & 0.20 \\
\hline
\end{tabular}

Alta Cima: 117 spp. (65 nativas y 52 exóticas); San José: 98 spp. (42 nativas y 56 exóticas). 
ambos géneros. Sin embargo, cuando se analiza lo relativo al conocimiento de especies nativas y exóticas, los habitantes de San José muestran índices de conocimiento (RQZ) más elevados sobre taxa nativos que en Alta Cima, para ambos sexos. En contraposición, en Alta Cima se encontraron los índices de conocimiento más elevados para taxa exóticos, en hombres y mujeres.

Hay diferencias significativas en cuanto a las especies utilizadas por hombres y mujeres en ambas comunidades (Tabla 5).
DISCUSIÓN

Al relacionar el índice RQZ con los rangos de edad, los valores numéricos más altos se encuentran entre los grupos etarios de 41 a 50 años y de 61 y más, hecho que puede atribuirse a que estas personas tradicionalmente están ligadas al aprovechamiento del bosque, especialmente a la recolección de palmilla (Chamaedorea radicalis Mart.). Esto concuerda parcialmente con lo identificado en comunidades campesinas, donde a medida que aumenta la edad de los informantes aumenta la

Tabla 5. Prueba $t$ para dos muestras suponiendo varianzas iguales

\begin{tabular}{|c|c|c|c|}
\hline & Mujer & Hombre & \\
\hline Media & 20.666666667 & 28 & \\
\hline Varianza & 53.76470588 & 11.9047619 & \\
\hline Observaciones & 18 & 22 & \\
\hline Varianza agrupada & 30.63157895 & & \\
\hline $\begin{array}{l}\text { Diferencia hipotética de las } \\
\text { medias }\end{array}$ & 0 & & \\
\hline Grados de libertad & 38 & & \\
\hline Estadístico $\dagger$ & -4.169023388 & & \\
\hline$P(T<=t)$ una cola & $8.52381 \mathrm{E}-05^{*}$ & & \\
\hline Valor crítico de t (una cola) & 1.68595446 & & \\
\hline$P(T<=t)$ dos colas & $0.000170476^{*}$ & & \\
\hline \multirow[t]{2}{*}{ Valor crítico de $t$ (dos colas) } & 2.024394164 & & \\
\hline & Mujer & Hombre & \\
\hline Media & 20.666666667 & Media & 28 \\
\hline Error típico & 1.728273157 & Error típico & 0.735612 \\
\hline Mediana & 22 & Mediana & 28 \\
\hline Moda & 26 & Moda & 28 \\
\hline Desviación estándar & 7.332442014 & Desviación estándar & 3.450328 \\
\hline Varianza de la muestra & 53.76470588 & Varianza de la muestra & 11.90476 \\
\hline Curtosis & -1.458569948 & Curtosis & 0.195241 \\
\hline Coeficiente de asimetría & -0.274513672 & $\begin{array}{l}\text { Coeficiente de } \\
\text { asimetría }\end{array}$ & -0.26015 \\
\hline Rango & 21 & Rango & 15 \\
\hline Mínimo & 9 & Mínimo & 20 \\
\hline Máximo & 30 & Máximo & 35 \\
\hline Suma & 372 & Suma & 616 \\
\hline Cuenta & 18 & Cuenta & 22 \\
\hline
\end{tabular}

*Si hay significancia, hay diferencias entre spp. utilizadas por $\mathrm{H}$ y $\mathrm{M}$. 
riqueza de conocimiento, y son los ancianos quienes más valoran y conocen las plantas (León Suquilanda et al., 2006; Ramos Hernández et al., 2007; Morales et al., 2011; Mercado, 2013).

Es importante resaltar que en los grupos etarios de jóvenes se mantienen altos valores de RQZ, lo cual contrasta con lo encontrado por autores como León Suquilanda et al. (2006) y Morales et al. (2011), quienes evidencian la pérdida parcial del conocimiento tradicional entre jóvenes campesinos por aculturación, migración y nula transmisión de información. Cuando se habla de RQZ a nivel de género no hay coincidencia; en Alta Cima los hombres conocen más que las mujeres, en San José en algunos casos las mujeres son las que poseen mayor conocimiento, $v$. gr. en plantas medicinales, alimentos, así como magicorreligiosas (Toscano González, 2006; Mercado, 2013; Bautista Cifuentes, 2015), al igual que los chamanes o curanderos (McDaniel y Alley, 2005). En otros casos son los hombres quienes más conocen de plantas, v. gr. cuando se trata de plantas forestales (madera, postes, vigas, no maderables o para aserrío) (León Suquilanda et al., 2006).

Con respecto al conocimiento de plantas nativas vs. exóticas, San José muestra RQZ más elevados para especies nativas y Alta Cima para especies exóticas; tanto para hombres como para mujeres. Algunos autores cuestionan la alta incidencia de plantas exóticas en el RQZ, atribuyen su predominancia como parte de un proceso de aculturación y un peligro para la permanencia del conocimiento tradicional; sin embargo, Bennet y Prance (2000) lo ven como el resultado de una estrategia de diversificación con tal de que esta incursión de nuevas especies y prácticas foráneas no sustituyan o desplacen a las nativas o redunden en homogeneización de las prácticas de manejo (McDaniel y Alley, 2005).

\section{CONCLUSIONES}

En la riqueza de conocimiento sobre plantas, la primera parte de la hipótesis no se comprueba al observarse un nivel de conocimiento homogéneo en los diferentes grupos etarios, sólo en la comunidad de Alta Cima existe una diferencia significativa en el grupo etario de 41 a 50 años. La segunda parte de la hipótesis que dice que las mujeres son quienes más conocen sobre plantas, tampoco se comprobó, ya que en una de las comunidades fueron los hombres (Alta Cima) y en la otra (San José), los valores son iguales. Este estudio permitió comprobar que el conocimiento etnobotánico se encuentra disperso entre los diferentes grupos de edad, donde cada grupo etario es poseedor de una pequeña porción de los conocimientos sobre plantas útiles en el bosque de niebla de la RBEC. Aquí las estrategias de vida diversificada mantienen en estas comunidades un sistema de conocimiento más complejo y especializado, lo cual es importante para la permanencia y subsistencia de dichos grupos campesinos que deben ser considerados en el diseño y desarrollo de modelos de manejo de recursos vegetales adaptativos con base en el conocimiento de los pobladores locales.

\section{LITERATURA CITADA}

- ALEXIADES, M. N. Standard techniques for collecting and preparing herbarium specimens. En M. N. ALEXIADES (Ed.), Selected guidelines for ethnobotanical research: a field manual. Advances in Economic Botany 10 (pp. 99-126). New York, US: The New York Botanical Garden, 1996.

- ARIAS, G. J. C. y CÁRDENAS LÓPEZ, D. Manual de identificación, selección y evaluación de oferta de productos forestales no maderables. Bogotá, Colombia: Instituto Amazónico de Investigaciones Científicas-Sinchi, 32 pp., 2007.

- BAUTISTA CIFUENTES, R. W. Etnobotánica de plantas medicinales y alimenticias nativas de Mesoamérica, en diez comunidades del Municipio de San Cristóbal Cucho, Departamento de San Carlos, Guatemala. Tesis de Doctorado. Guatemala: Universidad de San Carlos de Guatemala, 141 pp., 2015.
- BENNET, B. C. y PRANCE, G. T. Introduced plants in the indigenous pharmacopoeia of Northern South America. Economic Botany, 54(1): 90-102, 2000.

- BeRRONES BENITEZ, E. y MEDELLín MORALES, S. G. El uso de las plantas en la gastronomía de la Huasteca Tamaulipeca. Cd. Victoria, Tamaulipas, México: Instituto Tamaulipeco para la Cultura y las Artes, 149 pp., 2007.

- GARZA, M. et al. Los sistemas de producción en la vertiente semiárida. En G. SÁNCHEZ RAMOS, P. REYES y R. DIRZO (Eds.). Historia Natural de la Reserva de la Biósfera El Cielo, Tamaulipas, México (pp. 619-624). Cd. Victoria, Tamaulipas, México: Universidad Autónoma de Tamaulipas, 2005. 
- Germosen ROBINEAU, L. Hacia una farmacopea caribeña. Santo Domingo, República Dominicana: Edición TRAMIL 7 Enda-Caribe-UAG-Universidad de Antioquia, 75 pp., 1995.

- GOBIERNO DE TAMAULIPAS y THE NATURE CONSERVANCY. Plan de Conservación de la Reserva de la Biósfera El Cielo, Tamaulipas. Cd. Victoria, Tamaulipas, México: Autor, 126 pp., 2011.

- GONZÁLEZ ROMO, C. y GISPERT, M. Etnobotánica de los solares. En G. SÁNCHEZ RAMOS, P. REYES y R. DIRZO (Eds.), Historia Natural de la Reserva de la Biósfera El Cielo, Tamaulipas, México (pp. 579-590). Cd. Victoria, Tamaulipas, México: Universidad Autónoma de Tamaulipas, 2005.

- HERNÁNDEZ SANDOVAL, L. et al. Plantas útiles de Tamaulipas. Anales del Instituto de Biología de la Universidad Nacional Autónoma de México, Serie Botánica, 62(1): 1-38, 1991.

- HERSCH MARTíNEZ, P. y GONZÁLEZ CHÉVEZ, L. Investigación participativa en etnobotánica. Algunos procedimientos coadyuvantes en ella. Dimensión Antropológica, 8(septiembrediciembre): 129-153, 1996.

- HOFFMAN, B. y GALLAHER, T. Importance indices in ethnobotany. Ethnobotany Resources \& Applications, 5, 201218, 2007

- LARA, M. Estudio preliminar de las especies vegetales visitadas por Apis mellifera en la Reserva de la Biósfera El Cielo. Revista Biotam, 1(1): 14-18, 1989.

- LEÓN SUQUILANDA, L. M. et al. Floristic composition, structure, endemic \& ethnobotany in the native forest "El Colorado", in Puyango, Province of Loja. Lyonia, 10(2): 105-115, 2006.

- MCDANIEL, J. y ALLEY, K. Connecting local environmental knowledge and land use practices: a human ecosystem approach to urbanization in West Georgia. Urban Ecosystems Netherlands, 8, 23-38, 2005.
- medellín morales, S. y OSORIO, H. Manual de medicina veterinaria tradicional en la Huasteca Tamaulipeca. Cd. Victoria, Tamaulipas, México: Instituto Tamaulipeco para la Cultura y las Artes, 72 pp., 2007.

- Mercado, A. Estudio de las plantas medicinales usadas por los cuicatecos en la localidad de Santos Reyes Papalo, Cuicatlán, Oax. Tesis Biología. México: UNAM, 159 pp., 2013.

- MORA LÓPEZ, J. L. y MEDELLÍN MORALES, S. Los núcleos campesinos de la Reserva de la Biósfera El Cielo: zaaliados en la conservación? Revista Biotam, 4(2): 13-40, 1991.

- MORA Olivo, A. et al. Las plantas forrajeras. En G. SÁNCHEZ RAMOS, P. REYES y R. DIRZO (Eds.), Historia Natural de la Reserva de la Biósfera El Cielo, Tamaulipas, México (pp. 610618). Cd. Victoria, Tamaulipas, México: Universidad Autónoma de Tamaulipas, 2005.

- MORALES, R. et al. Biodiversidad y Etnobotánica en España. Memorias Real Sociedad Española de Historia Natural, 912 ép.): 157-207, 2011.

- PÉREZ QUILANTÁN, L. et al. Las plantas comestibles silvestres. En G. SÁNCHEZ RAMOS, P. REYES y R. DIRZO (Eds.), Historia Natural de la Reserva de la Biósfera El Cielo, Tamaulipas, México (pp. 604-609). Cd. Victoria, Tamaulipas, México: Universidad Autónoma de Tamaulipas, 2005.

- RAMOS hERNÁNDEZ, M. et al. Etnobotánica y ecología de plantas utilizadas por tres curanderos contra la mordedura de serpiente en la región de Acayucan, Veracruz, México. Boletín de la Sociedad Botánica de México, 81, 89-100, 2007.

- STRINGER, L. C. et al. Unpacking "participation" in the adaptive management of social-ecological systems: a critical review. Ecology and Society, Nova Scotia, vol. 11 (2): 39, 2006.

- tOSCANO GONZÁlEZ, J. Y. Uso tradicional de plantas medicinales en la vereda San Isidro, Municipio de San José de Pare-Boyacá: un estudio preliminar usando técnicas cuantitativas. Acta Biológica Colombiana, 11 (2): 137-146, 2006. 